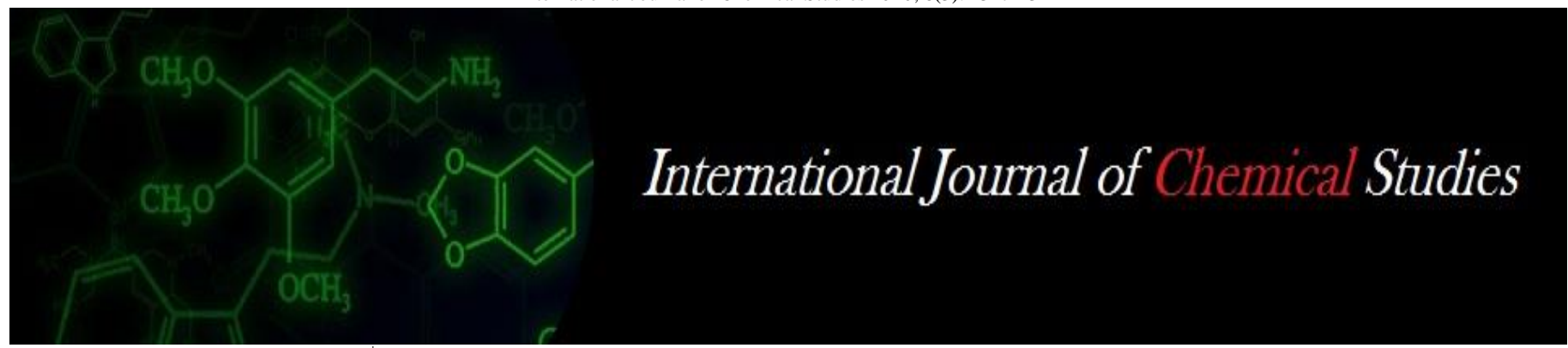

P-ISSN: 2349-8528

E-ISSN: 2321-4902

www.chemijournal.com

IJCS 2020; 8(3): 1317-1321

(C) 2020 IJCS

Received: 10-03-2020

Accepted: 12-04-2020

V Sravani

Department of Vegetable

Science, Navsari Agricultural

University, Navsari, Gujarat,

India

SN Saravaiya

Department of Vegetable

Science, Navsari Agricultural

University, Navsari, Gujarat,

India

DR Bhanderi

Department of Vegetable

Science, Navsari Agricultural

University, Navsari, Gujarat,

India

\section{Dev Raj}

Department of Vegetable

Science, Navsari Agricultural

University, Navsari, Gujarat,

India

\section{Himani B Patel}

Department of Vegetable

Science, Navsari Agricultural

University, Navsari, Gujarat,

India

\section{JM Vash}

Department of Vegetable

Science, Navsari Agricultural University, Navsari, Gujarat, India

Corresponding Author: V Sravani

Department of Vegetable Science, Navsari Agricultural University, Navsari, Gujarat, India

\section{Effect of plant growth regulators on biochemical analysis of onion (Allium cepa)}

\author{
V Sravani, SN Saravaiya, DR Bhanderi, Dev Raj, Himani B Patel and JM \\ Vashi
}

DOI: https://doi.org/10.22271/chemi.2020.v8.i3r.9381

\begin{abstract}
A field experiment was carried out during Rabi 2018 and 2019 at RHRS, Navsari Agricultural University, Navsari, Gujarat to study the effect of different plant growth regulators like $\mathrm{GA}_{3}$ and NAA on biochemical parameters of onion. The treatment $\mathrm{T}_{1}\left(\mathrm{GA}_{3} 25 \mathrm{mg} \mathrm{l}^{-1}\right)$ recorded highest chlorophyll content of leaves 45 DATP (1.06 mg $\left.100 \mathrm{~g} \mathrm{~g}^{-1}\right), 60$ DATP (2.64 mg $\left.100 \mathrm{~g}^{-1}\right), 90$ DATP (2.32 mg $100 \mathrm{~g} \mathrm{~g}^{-1}$ ), ascorbic acid $\left(10.45 \mathrm{mg} 100 \mathrm{~g}^{-1}\right)$, phenols $\left(61.77 \mathrm{mg} 100 \mathrm{~g} \mathrm{~g}^{-1}\right)$ and proteins $\left(1072.54 \mathrm{mg} 100 \mathrm{~g}^{-1}\right)$ and found significant. The moisture content $\left(83.98 \%\right.$ ) under T6 (NAA $75 \mathrm{mg} \mathrm{l}^{-1}$ ) were found significant.
\end{abstract}

Keywords: Plant growth regulators, $\mathrm{GA}_{3}$, NAA, onion etc.

\section{Introduction}

India is the world's second largest producer of vegetables (187.47 million tonnes) next only to China (Anonymous, 2019). Onion (Allium cepa L.) is an important and indispensable item in every kitchen as condiment cum vegetable in India. It is one of the important underground bulbous vegetable crops of Alliaceae family and is said to be native of Central Asia and Mediterranean region (Mc Collum, 1976). Plant growth regulators are organic compounds other than nutrients which in small amount promotes / inhibit or otherwise modify any physiological response in plant (Purohit, 2007) ${ }^{[9]}$. Plant bioregulators called as magic chemicals are new generation agrochemicals, when added in small quantity, modify the natural growth regulatory systems right from seed germination to senescence in several vegetable crops and also regulate and modify various physiological processes within the plant and they help to increase the yield (Weaver, 1972) ${ }^{[12]}$.

\section{Materials and methods}

The field experiment was carried out at the vegetable research scheme, Regional Horticultural Research Station of the Navsari Agricultural University, Navsari, Gujarat, India during Rabi 2018 and 2019 on cv. Gujarat Junagadh Red Onion 11 to investigate the response of plant bioregulators on growth parameters and plant growth analysis of onion. The experiment was conducted in Randomized Block Design (RBD) with three replications, which included 12 treatments namely, $\mathrm{T}_{1}$ : $\mathrm{GA}_{3} 25 \mathrm{mg} \mathrm{l}^{-1}, \mathrm{~T}_{2}$ : $\mathrm{GA}_{3} 50 \mathrm{mg} \mathrm{l}^{-1}, \mathrm{~T}_{3}: \mathrm{GA}_{3} 75 \mathrm{mg} \mathrm{l}^{-1}, \mathrm{~T}_{4}$ : NAA $25 \mathrm{mg} \mathrm{l}^{-}$ ${ }^{1}, \mathrm{~T}_{5}$ : NAA $50 \mathrm{mg} \mathrm{l}^{-1}, \mathrm{~T}_{6}$ : NAA $75 \mathrm{mg} \mathrm{l}^{-1}, \mathrm{~T}_{7}: \mathrm{GA}_{3} 25 \mathrm{mg} \mathrm{l}^{-1}+\mathrm{NAA}_{2} 25 \mathrm{mg} \mathrm{l}^{-1}, \mathrm{~T}_{8}$ : $\mathrm{GA}_{3} 25 \mathrm{mg}$ $\mathrm{l}^{-1}+\mathrm{NAA} 50 \mathrm{mg} \mathrm{l}^{-1}, \mathrm{~T}_{9}: \mathrm{GA}_{3} 25 \mathrm{mg} \mathrm{l}^{-1}+\mathrm{NAA} 75 \mathrm{mg} \mathrm{l}^{-1}, \mathrm{~T}_{10}: \mathrm{GA}_{3} 50 \mathrm{mg} \mathrm{l}^{-1}+\mathrm{NAA} 50 \mathrm{mg} \mathrm{l}^{-1}$, $\mathrm{T}_{11}$ : $\mathrm{GA}_{3} 75 \mathrm{mg} \mathrm{l}^{-1}+\mathrm{NAA} 75 \mathrm{mg} \mathrm{l}^{-1}$ and $\mathrm{T}_{12}$ : Control. The foliar sprays were made at 30 days after transplanting during morning hours to avoid the dehydration effect. For recording different observations, ten plants of onion from each net plot area were selected randomly and tagged with labels.

\section{Results}

Proteins (mg $\left.100 \mathrm{~g}^{-1}\right)$

Results of proteins in onion bulb under different treatments showed significant in pooled analysis. The maximum protein content $(1072.54 \mathrm{mg})$ observed with $\mathrm{T}_{1}\left(\mathrm{GA}_{3} 25 \mathrm{mg} \mathrm{l}^{-1}\right)$ followed by the treatment $\mathrm{T}_{10}\left(\mathrm{GA}_{3} 50 \mathrm{mg} \mathrm{l}^{-1}+\mathrm{NAA} 50 \mathrm{mg} \mathrm{l}^{-\mathbf{1}}\right)$. Whereas, $\mathrm{GA}_{3} 75 \mathrm{mg} \mathrm{l}^{-1}+$ NAA $75 \mathrm{mg} \mathrm{l}^{-1}\left(\mathrm{~T}_{11}\right)$ recorded minimum protein content $(866.82 \mathrm{mg})$. The interaction of year $\times$ 
treatment was found non-significant.

\section{Phenols (mg $100 \mathrm{~g}^{-1}$ )}

The results of percent total phenols under different treatments showed significant in pooled analysis. The maximum phenol content $(61.77 \mathrm{mg})$ observed with the treatment $\mathrm{T}_{1}\left(\mathrm{GA}_{3} 25\right.$ $\mathrm{mg} \mathrm{l}^{-1}$ ) which was significantly at par with $\mathrm{T}_{12}$. The minimum phenol content $(51.53 \mathrm{mg})$ observed with the treatment $\mathrm{T}_{3}$. The interaction of year $\times$ treatment was found non-significant.

\section{Chlorophyll content of leaves $\left(\mathrm{mg} 100 \mathrm{~g}^{-1}\right)$}

The results of chlorophyll content under different treatments found significant. In pooled analysis, the maximum chlorophyll content (1.06 mg; $2.64 \mathrm{mg}$ and $2.32 \mathrm{mg}$ ) at 45, 60 and 90 DATP respectively was recorded with the treatment $\mathrm{T}_{1}$ $\left(\mathrm{GA}_{3} 25 \mathrm{mg} \mathrm{l}^{-1}\right)$. The minimum chlorophyll content $(0.82 \mathrm{mg})$ was recorded with $\mathrm{T}_{12}$ (Control) at 45 DATP whereas, chlorophyll content $(2.33 \mathrm{mg}$ and $2.07 \mathrm{mg})$ at 60 and 90 DATP respectively, was observed with the treatment $\mathrm{T}_{3}\left(\mathrm{GA}_{3}\right.$ $\left.75 \mathrm{mg} \mathrm{l}^{-1}\right)$.

\section{Reducing sugar (\%)}

During the first season, the maximum percentage of reducing sugars $(5.21 \%)$ observed with $\mathrm{T}_{4}$ (NAA $25 \mathrm{mg} \mathrm{l}^{-1}$ ) which was significantly at par with the treatments $\mathrm{T}_{5}, \mathrm{~T}_{9}, \mathrm{~T}_{6}$ and $\mathrm{T}_{3}$. Whereas, the minimum reducing sugars (3.67\%) was registered with control $\left(\mathrm{T}_{12}\right)$. During the second season, the treatment $\mathrm{GA}_{3} 75 \mathrm{mg} \mathrm{l}^{-1}\left(\mathrm{~T}_{3}\right)$ was recorded maximum reducing sugars percentage $(6.62 \%)$. Whereas, $\mathrm{T}_{4}$ (NAA 25 $\mathrm{mg}^{-1}$ ) recorded minimum reducing sugars percentage $(4.15 \%)$. In pooled analysis, the results of reducing sugars under different treatments found non-significant.

\section{Total sugar (\%)}

During the first season, the maximum total sugar (6.94\%) was found with the treatment $\mathrm{T}_{10}\left(\mathrm{GA}_{3} 50 \mathrm{mg} \mathrm{l}^{-1}+\mathrm{NAA} 50 \mathrm{mg} \mathrm{l}^{-1}\right)$ which was significantly at par with the treatment $\mathrm{T}_{8}$. As well as, the minimum percent of total sugars $(5.06 \%)$ was found with the treatment control $\left(\mathrm{T}_{12}\right)$. During the second season, application of NAA $75 \mathrm{mg} \mathrm{l}^{-1}\left(\mathrm{~T}_{6}\right)$ recorded maximum total sugar $(8.65 \%)$ which was significantly at par with the treatment $\mathrm{T}_{9}$. Whereas, $\mathrm{GA}_{3} 25 \mathrm{mg} \mathrm{l}^{-1}\left(\mathrm{~T}_{1}\right)$ recorded minimum total sugars $(5.73 \%)$. Looking to the mean of pooled analysis, results of total sugars under different treatments was nonsignificant.

\section{Non- reducing sugar $(\%)$}

During the first season, maximum non-reducing sugars $(2.82 \%)$ found with the treatment $\mathrm{T}_{10}\left(\mathrm{GA}_{3} 50 \mathrm{mg} \mathrm{l}^{-1}+\mathrm{NAA}\right.$ $50 \mathrm{mg} \mathrm{l}^{-1}$ ) which was superior over other treatments followed by $\mathrm{T}_{8}$. Whereas, the minimum non-reducing sugars $(0.59 \%)$ was found with the treatment $\mathrm{T}_{4}$ (NAA $25 \mathrm{mg} \mathrm{l}^{-1}$ ). The significantly maximum non-reducing sugars $(4.76 \%)$ was observed with the treatment $\mathrm{T}_{6}$ (NAA $75 \mathrm{mg} \mathrm{l}^{-1}$ ) followed by $\mathrm{T}_{9}$ during the second season. Whereas, the minimum nonreducing sugars $(0.73 \%)$ was recorded with the treatment $\mathrm{T}_{3}$ $\left(\mathrm{GA}_{3} 75 \mathrm{mg} \mathrm{l}^{-1}\right)$. The results of percent non-reducing sugars under different treatments showed non-significant in pooled analysis. The interaction of year $\times$ treatment was found nonsignificant.

\section{TSS (\%)}

In pooled analysis, the results of TSS under different treatments showed non-significant. The interaction of year $x$ treatment was found significant.

\section{Moisture content (\%)}

Looking to the mean of pooled analysis, the maximum moisture content $\left(83.98 \%\right.$ ) was obtained with $\mathrm{T}_{6}$ (NAA $75 \mathrm{mg}$ $1^{-1}$ ) which was significantly at par with the treatments $\mathrm{T}_{5}, \mathrm{~T}_{8}$, $\mathrm{T}_{11}, \mathrm{~T} 1$ and $\mathrm{T}_{10}$. The minimum moisture content $(78.81 \%)$ was noted in control $\left(\mathrm{T}_{12}\right)$. The interaction of year $\times$ treatment was found non-significant.

\section{Ascorbic acid content ( $\left.\mathrm{mg} 100 \mathrm{~g}^{-1}\right)$}

The results of ascorbic acid under different treatments showed significant in pooled analysis. The maximum ascorbic acid content $(10.45 \mathrm{mg})$ was observed with the treatment $\mathrm{T}_{1}\left(\mathrm{GA}_{3}\right.$ $25 \mathrm{mg} \mathrm{l}^{-1}$ ) which was significantly superior followed by $\mathrm{T}_{10}$. The minimum ascorbic acid content $(8.16 \mathrm{mg})$ was observed with the treatment control $\left(\mathrm{T}_{12}\right)$. The interaction of year $\times$ treatment was found non-significant.

\section{Bulb pH}

The significantly highest bulb $\mathrm{pH}$ (4.78) was found with the application of $\mathrm{GA}_{3} 75 \mathrm{mg} \mathrm{l}^{-1}+\mathrm{NAA} 75 \mathrm{mg} \mathrm{l}^{-1}\left(\mathrm{~T}_{11}\right)$ which was significantly at par with the treatments $T_{3}, T_{12}, T_{6}, T_{9}, T_{8}$, $\mathrm{T}_{5}, \mathrm{~T}_{10}, \mathrm{~T}_{2}$ and $\mathrm{T}_{4}$ in first season. Whereas the lowest bulb $\mathrm{pH}$ (4.48) was obtained in $\mathrm{T}_{1}\left(\mathrm{GA}_{3} 75 \mathrm{mg} \mathrm{l}^{-1}\right)$. During the second season, the maximum bulb $\mathrm{pH}$ (4.77) was observed with $\mathrm{T}_{6}$ (NAA $75 \mathrm{mg} \mathrm{l}^{-1}$ ) which was significantly at par with the other treatments $\mathrm{T}_{5}, \mathrm{~T}_{3}, \mathrm{~T}_{11}, \mathrm{~T}_{10}, \mathrm{~T}_{7}, \mathrm{~T}_{1}, \mathrm{~T}_{2}$ and $\mathrm{T}_{4}$. The minimum bulb $\mathrm{pH}$ (4.38) was observed in control $\left(\mathrm{T}_{12}\right)$. The results of bulb $\mathrm{pH}$ under different treatments varied from 4.55 to 4.76 but it was non-significant. The interaction of year $\times$ treatment was found significant

\section{Discussion}

Chlorophyll-a and Chlorophyll-b were increased with $\mathrm{GA}_{3}$ treatments in Andrographis paniculata (Gomatinayagam et al., 2009). The results indicated that there was increase in chlorophyll content due to foliar application of gibberellic acid $\left(\mathrm{GA}_{3}\right)$ induces enhancement of ultra structural morphogenesis of plastids, which coupled with retention of chlorophyll, delay plant senescence (Arteca, 1996; Ouzounidu and Ilias, 2005; Shah et al., 2007; Ouzounidu et al., 2011) ${ }^{[1,6 \text {, }}$ 10]. This increase undoubtedly might have helped to improve the photosynthetic efficiency. The application of growth regulators may prove beneficial for improvement of growth and productivity of economically important vegetable crop onion. The plants under the influence of $\mathrm{GA}_{3}$ was found with increased soluble carbohydrates, ascorbic acid content in tomato. Growth regulators improve the quality parameters of the onion bulbs due to enhanced physiological activity. The increase in TSS may be accounted due to the hydrolysis of polysaccharides. Conversion of organic acids in to soluble sugars and enhanced solubilisation of insoluble starch and pectin present in the cell wall and middle lamella. The increase in TSS content due to growth regulators found from the results of several workers (Tiwari et al., 2003; and Patel et al., 2010) ${ }^{[11,8]}$ and Govind et al. $2015^{[4]}$ in garlic. The production of ascorbic acid content seems to be enhanced under $\mathrm{GA}_{3}$. The increase in ascorbic acid content may be due to vitamin can be synthesized in plant by the process involves the conversion of hexose sugar mainly glucose and galactose into ascorbic acid. The results are in conformity with Ouzounidou et al. (2011) ${ }^{[5]}$ in onion, Chaudhary et al. (2006) ${ }^{[2]}$ and Ouzounidu et al. (2010) ${ }^{[7]}$ in chilli and pepper. The moisture content showed significant results with NAA. Similar results with Govind et al. $(2015)^{[4]}$ in garlic. 


\section{Acknowledgements}

I wish to express my sincere gratitude to Dr. S.N. Saravaiya, Associate Professor for providing an opportunity to do my project work in RHRS, Navsari Agricultural University, Gujarat during the year 2018 - 2019.

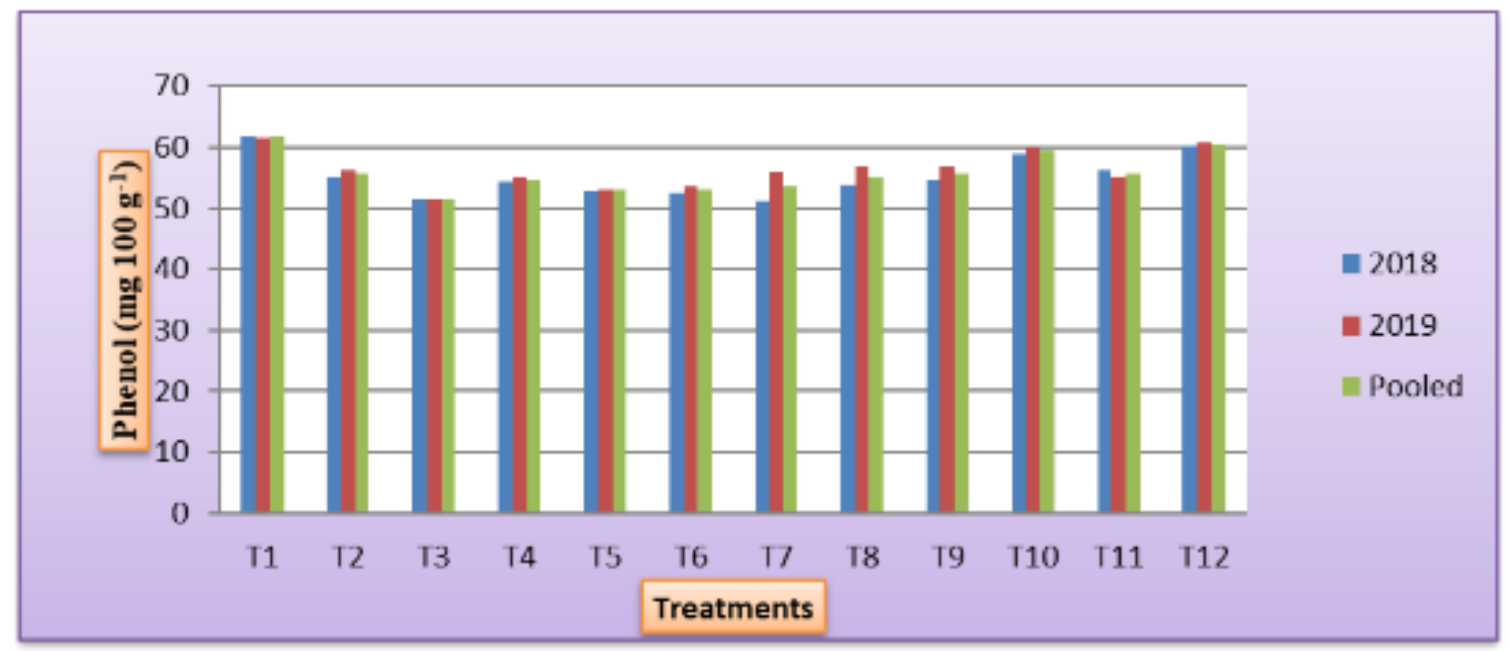

Fig 1: Effect of different treatments on phenol content $\left(\mathrm{mg} 100 \mathrm{~g}^{-1}\right)$ in onion

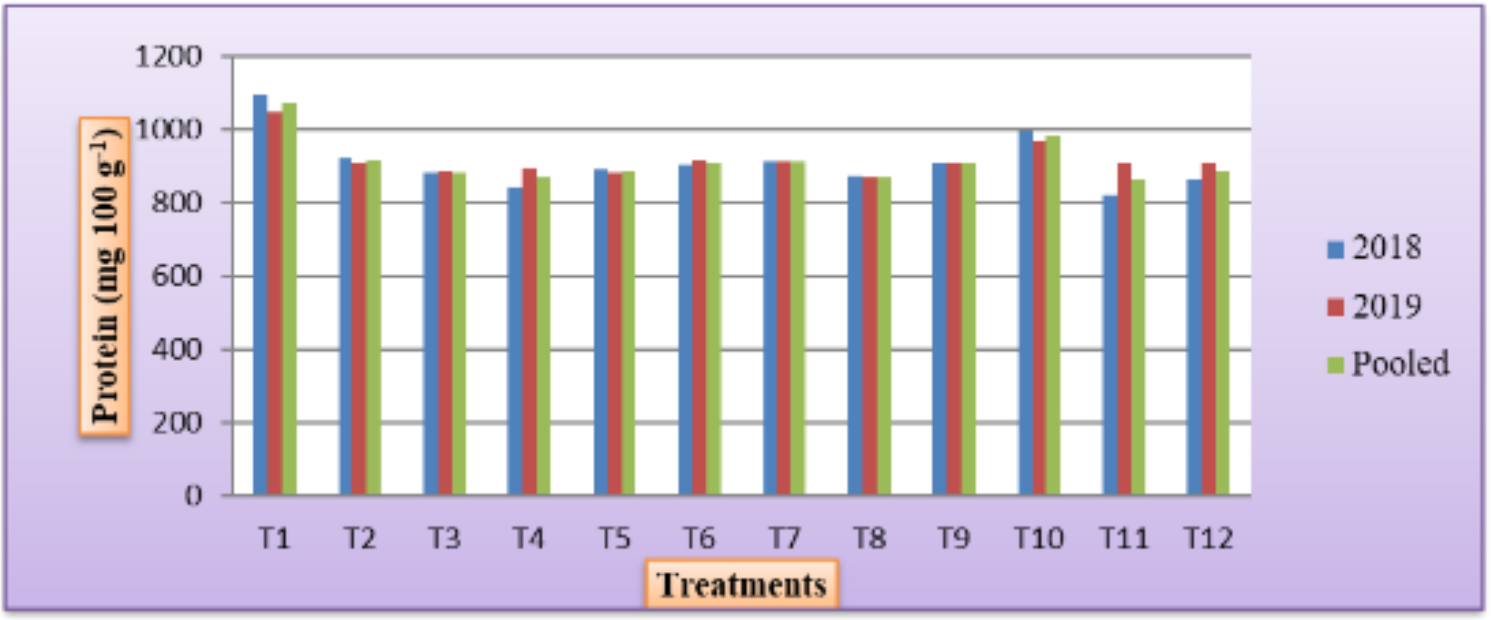

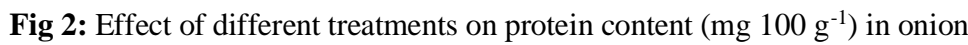

Table 1: Effect of different treatments on chlorophyll content of leaves $\left(\mathrm{mg} 100 \mathrm{~g}^{-1}\right)$ of onion

\begin{tabular}{|c|c|c|c|c|c|c|c|c|c|}
\hline \multirow{3}{*}{ Treatments } & \multicolumn{9}{|c|}{ Chlorophyll content of leaves $\left(\mathrm{mg}^{\left.100 \mathrm{~g}^{-1}\right)}\right.$} \\
\hline & \multicolumn{3}{|c|}{45 DATP } & \multicolumn{3}{|c|}{ 60 DATP } & \multicolumn{3}{|c|}{90 DATP } \\
\hline & 2018 & 2019 & Pooled & 2018 & 2019 & Pooled & 2018 & 2019 & Pooled \\
\hline $\mathrm{T}_{1}: \mathrm{GA}_{3} 25 \mathrm{mg} \mathrm{l}^{-1}$ & 1.08 & 1.04 & 1.06 & 2.55 & 2.73 & 2.64 & 2.22 & 2.41 & 2.32 \\
\hline $\mathrm{T}_{2}: \mathrm{GA}_{3} 50 \mathrm{mg} \mathrm{l}^{-1}$ & 0.92 & 0.91 & 0.92 & 2.15 & 2.24 & 2.20 & 1.84 & 2.03 & 1.93 \\
\hline $\mathrm{T}_{3}: \mathrm{GA}_{3} 75 \mathrm{mg} \mathrm{l}^{-1}$ & 0.85 & 0.86 & 0.86 & 2.21 & 2.45 & 2.33 & 1.99 & 2.15 & 2.07 \\
\hline $\mathrm{T}_{4}$ : NAA $25 \mathrm{mg} \mathrm{l}^{-1}$ & 0.85 & 0.85 & 0.85 & 1.56 & 1.57 & 1.57 & 1.31 & 1.43 & 1.37 \\
\hline $\mathrm{T}_{5}:$ NAA $50 \mathrm{mg} \mathrm{l}^{-1}$ & 0.89 & 0.94 & 0.92 & 2.00 & 2.30 & 2.15 & 1.71 & 1.73 & 1.72 \\
\hline $\mathrm{T}_{6}:$ NAA $75 \mathrm{mg} \mathrm{l}^{-1}$ & 0.87 & 0.89 & 0.88 & 2.22 & 2.40 & 2.31 & 1.91 & 2.12 & 2.02 \\
\hline $\mathrm{T}_{7}: \mathrm{GA}_{3} 25 \mathrm{mg} \mathrm{l}^{-1}+\mathrm{NAA} 25 \mathrm{mg} \mathrm{l}^{-1}$ & 0.91 & 0.86 & 0.89 & 2.28 & 2.23 & 2.25 & 1.95 & 2.16 & 2.06 \\
\hline $\mathrm{T}_{8}: \mathrm{GA}_{3} 25 \mathrm{mg} \mathrm{l}^{-1}+\mathrm{NAA} 50 \mathrm{mg} \mathrm{l}^{-1}$ & 0.94 & 0.95 & 0.94 & 2.19 & 2.32 & 2.25 & 1.90 & 2.18 & 2.04 \\
\hline $\mathrm{T}_{9}: \mathrm{GA}_{3} 25 \mathrm{mg} \mathrm{l}^{-1}+\mathrm{NAA} 75 \mathrm{mg} \mathrm{l}^{-1}$ & 0.90 & 0.92 & 0.91 & 2.09 & 2.17 & 2.13 & 1.73 & 1.86 & 1.80 \\
\hline $\mathrm{T}_{10}: \mathrm{GA}_{3} 50 \mathrm{mg} \mathrm{l}^{-1}+\mathrm{NAA} 50 \mathrm{mg} \mathrm{l}^{-1}$ & 0.93 & 0.96 & 0.95 & 2.29 & 2.37 & 2.33 & 1.88 & 2.04 & 1.96 \\
\hline $\mathrm{T}_{11}: \mathrm{GA}_{3} 75 \mathrm{mg} \mathrm{l}^{-1}+\mathrm{NAA} 75 \mathrm{mg} \mathrm{l}^{-1}$ & 0.86 & 0.82 & 0.84 & 1.73 & 1.87 & 1.80 & 1.43 & 1.49 & 1.46 \\
\hline $\mathrm{T}_{12}:$ Control & 0.84 & 0.80 & 0.82 & 1.59 & 1.76 & 1.68 & 1.35 & 1.46 & 1.41 \\
\hline Year Mean & 0.90 & 0.90 & 0.90 & 2.07 & 2.20 & 2.14 & 1.77 & 1.92 & 1.85 \\
\hline S. Em. \pm & 0.02 & 0.03 & 0.01 & 0.05 & 0.06 & 0.04 & 0.05 & 0.05 & 0.03 \\
\hline C.D. at $5 \%$ & 0.06 & 0.08 & 0.05 & 0.16 & 0.18 & 0.12 & 0.13 & 0.14 & 0.10 \\
\hline C.V.\% & 3.88 & 4.94 & 4.44 & 4.49 & 4.86 & 4.69 & 4.46 & 4.32 & 4.39 \\
\hline \multicolumn{3}{|l|}{ YT: S. Em. \pm} & 0.02 & & & 0.06 & & & 0.05 \\
\hline YT: C. D. at 5\% & & & $\mathrm{NS}$ & & & $\mathrm{NS}$ & & & NS \\
\hline
\end{tabular}


Table 2: Effect of different treatments on reducing sugar, total sugar and non-reducing sugar of onion

\begin{tabular}{|c|c|c|c|c|c|c|c|c|c|}
\hline \multirow{2}{*}{ Treatments } & \multicolumn{3}{|c|}{ Reducing sugar (\%) } & \multicolumn{3}{|c|}{ Total sugar (\%) } & \multicolumn{3}{|c|}{ Non-reducing sugar (\%) } \\
\hline & 2018 & 2019 & Pooled & 2018 & 2019 & Pooled & 2018 & 2019 & Pooled \\
\hline $\mathrm{T}_{1}: \mathrm{GA}_{3} 25 \mathrm{mg} \mathrm{l}^{-1}$ & 4.67 & 4.62 & 4.65 & 5.69 & 5.73 & 5.71 & 1.02 & 1.11 & 1.07 \\
\hline $\mathrm{T}_{2}: \mathrm{GA}_{3} 50 \mathrm{mg} \mathrm{l}^{-1}$ & 4.26 & 4.63 & 4.44 & 5.60 & 5.95 & 5.78 & 1.34 & 1.32 & 1.33 \\
\hline $\mathrm{T}_{3}: \mathrm{GA}_{3} 75 \mathrm{mg} \mathrm{l}^{-1}$ & 4.84 & 6.62 & 5.73 & 5.65 & 7.35 & 6.50 & 0.81 & 0.73 & 0.77 \\
\hline $\mathrm{T}_{4}: \mathrm{NAA} 25 \mathrm{mg} \mathrm{l}^{-1}$ & 5.21 & 4.15 & 4.68 & 5.80 & 7.02 & 6.41 & 0.59 & 3.00 & 1.80 \\
\hline $\mathrm{T}_{5}:$ NAA $50 \mathrm{mg} \mathrm{l}^{-1}$ & 4.98 & 5.24 & 5.11 & 6.26 & 7.55 & 6.90 & 1.28 & 2.31 & 1.80 \\
\hline $\mathrm{T}_{6}: \mathrm{NAA} 75 \mathrm{mg} \mathrm{l}^{-1}$ & 4.85 & 3.89 & 4.37 & 5.55 & 8.65 & 7.10 & 0.70 & 4.76 & 2.73 \\
\hline $\mathrm{T}_{7}: \mathrm{GA}_{3} 25 \mathrm{mg} \mathrm{l}^{-1}+\mathrm{NAA} 25 \mathrm{mg} \mathrm{l}^{-1}$ & 4.03 & 5.15 & 4.59 & 5.15 & 6.52 & 5.84 & 1.12 & 1.37 & 1.25 \\
\hline $\mathrm{T}_{8}: \mathrm{GA}_{3} 25 \mathrm{mg} \mathrm{l}^{-1}+\mathrm{NAA} 50 \mathrm{mg} \mathrm{l}^{-1}$ & 4.09 & 5.09 & 4.59 & 6.60 & 6.93 & 6.77 & 2.51 & 1.84 & 2.18 \\
\hline $\mathrm{T}_{9}: \mathrm{GA}_{3} 25 \mathrm{mg} \mathrm{l}^{-1}+\mathrm{NAA} 75 \mathrm{mg} \mathrm{l}^{-1}$ & 4.87 & 4.05 & 4.46 & 5.94 & 8.46 & 7.20 & 1.07 & 4.41 & 2.74 \\
\hline $\mathrm{T}_{10}: \mathrm{GA}_{3} 50 \mathrm{mg} \mathrm{l}^{-1}+\mathrm{NAA} 50 \mathrm{mg} \mathrm{l}^{-1}$ & 4.11 & 5.33 & 4.72 & 6.94 & 6.97 & 6.95 & 2.82 & 1.67 & 2.25 \\
\hline $\mathrm{T}_{11}: \mathrm{GA}_{3} 75 \mathrm{mg} \mathrm{l}^{-1}+\mathrm{NAA} 75 \mathrm{mg} \mathrm{l}^{-1}$ & 4.08 & 4.59 & 4.34 & 5.72 & 5.91 & 5.82 & 1.64 & 1.35 & 1.49 \\
\hline $\mathrm{T}_{12:}$ Control & 3.67 & 4.88 & 4.28 & 5.06 & 7.07 & 6.07 & 1.39 & 2.20 & 1.79 \\
\hline Year Mean & 4.47 & 4.85 & 4.66 & 5.83 & 7.01 & 6.42 & 1.36 & 2.17 & 1.76 \\
\hline S. Em. \pm & 0.13 & 0.13 & 0.47 & 0.14 & 0.20 & 0.51 & 0.03 & 0.06 & 0.81 \\
\hline C.D. at $5 \%$ & 0.37 & 0.39 & NS & 0.40 & 0.59 & NS & 0.09 & 0.17 & NS \\
\hline C.V.\% & 4.86 & 4.70 & 4.78 & 4.06 & 4.96 & 0.17 & 3.90 & 4.72 & 4.62 \\
\hline \multicolumn{3}{|l|}{ YT: S. Em. \pm} & 0.13 & & & 0.49 & & & 0.05 \\
\hline YT: C. D. at $5 \%$ & & & 0.37 & & & 4.63 & & & 0.14 \\
\hline
\end{tabular}

Table 3: Effect of different treatments on TSS (\%) and moisture content (\%) of onion

\begin{tabular}{|c|c|c|c|c|c|c|}
\hline \multirow{2}{*}{ Treatments } & \multicolumn{3}{|c|}{ TSS (\%) } & \multicolumn{3}{|c|}{ Moisture content (\%) } \\
\hline & 2018 & 2019 & Pooled & 2018 & 2019 & Pooled \\
\hline $\mathrm{T}_{1}: \mathrm{GA}_{3} 25 \mathrm{mg} \mathrm{l}^{-1}$ & 15.20 & 16.17 & 15.68 & 82.10 & 81.09 & 81.60 \\
\hline $\mathrm{T}_{2}: \mathrm{GA}_{3} 50 \mathrm{mg} \mathrm{l}^{-1}$ & 14.17 & 12.67 & 13.42 & 82.73 & 79.15 & 80.94 \\
\hline $\mathrm{T}_{3}: \mathrm{GA}_{3} 75 \mathrm{mg} \mathrm{l}^{-1}$ & 13.43 & 14.00 & 13.72 & 83.49 & 79.50 & 81.50 \\
\hline $\mathrm{T}_{4}$ : NAA $25 \mathrm{mg} \mathrm{l}^{-1}$ & 14.70 & 15.00 & 14.85 & 79.26 & 78.90 & 79.08 \\
\hline $\mathrm{T}_{5}:$ NAA $50 \mathrm{mg} \mathrm{l}^{-1}$ & 14.37 & 15.00 & 14.68 & 84.36 & 82.62 & 83.49 \\
\hline $\mathrm{T}_{6}: \mathrm{NAA} 75 \mathrm{mg} \mathrm{l}^{-1}$ & 14.40 & 15.58 & 14.99 & 84.89 & 83.07 & 83.98 \\
\hline $\mathrm{T}_{7}: \mathrm{GA}_{3} 25 \mathrm{mg} \mathrm{l}^{-1}+\mathrm{NAA} 25 \mathrm{mg} \mathrm{l}^{-1}$ & 14.87 & 15.33 & 15.10 & 78.81 & 81.03 & 79.92 \\
\hline $\mathrm{T}_{8}: \mathrm{GA}_{3} 25 \mathrm{mg} \mathrm{l}^{-1}+$ NAA $50 \mathrm{mg} \mathrm{l}^{-1}$ & 13.87 & 15.75 & 14.81 & 82.68 & 82.71 & 82.69 \\
\hline $\mathrm{T}_{9}: \mathrm{GA}_{3} 25 \mathrm{mg} \mathrm{l}^{-1}+\mathrm{NAA} 75 \mathrm{mg} \mathrm{l}^{-1}$ & 15.03 & 15.33 & 15.18 & 80.86 & 80.56 & 80.71 \\
\hline $\mathrm{T}_{10}: \mathrm{GA}_{3} 50 \mathrm{mg} \mathrm{l}^{-1}+\mathrm{NAA} 50 \mathrm{mg} \mathrm{l}^{-1}$ & 13.87 & 15.30 & 14.58 & 82.03 & 81.14 & 81.59 \\
\hline $\mathrm{T}_{11}: \mathrm{GA}_{3} 75 \mathrm{mg} \mathrm{l}^{-1}+\mathrm{NAA} 75 \mathrm{mg} \mathrm{l}^{-1}$ & 14.73 & 14.83 & 14.78 & 81.77 & 82.97 & 82.37 \\
\hline $\mathrm{T}_{12}:$ Control & 13.73 & 15.00 & 14.37 & 79.16 & 78.47 & 78.81 \\
\hline Year Mean & 14.36 & 15.00 & 14.68 & 81.85 & 80.93 & 81.39 \\
\hline S. Em. \pm & 0.31 & 0.38 & 0.43 & 1.33 & 1.09 & 0.86 \\
\hline C.D. at $5 \%$ & 0.92 & 1.12 & NS & 3.89 & 3.18 & 2.44 \\
\hline C.V.\% & 3.80 & 4.42 & 4.14 & 2.81 & 2.32 & 2.58 \\
\hline \multicolumn{3}{|l|}{ YT: S. Em. \pm} & 0.35 & & & 1.21 \\
\hline \multicolumn{3}{|l|}{ YT: C. D. at $5 \%$} & 1.00 & & & $\mathrm{NS}$ \\
\hline
\end{tabular}

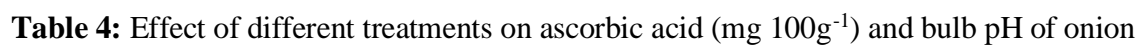

\begin{tabular}{|c|c|c|c|c|c|c|}
\hline \multirow{2}{*}{ Treatments } & \multicolumn{3}{|c|}{ Ascorbic acid (mg $\left.100 \mathrm{~g}^{-1}\right)$} & \multicolumn{3}{|c|}{ Bulb pH } \\
\hline & 2018 & 2019 & Pooled & 2018 & 2019 & Pooled \\
\hline $\mathrm{T}_{1}: \mathrm{GA}_{3} 25 \mathrm{mg} \mathrm{l}^{-1}$ & 10.41 & 10.50 & 10.45 & 4.48 & 4.61 & 4.55 \\
\hline $\mathrm{T}_{2}: \mathrm{GA}_{3} 50 \mathrm{mg} \mathrm{l}^{-1}$ & 8.40 & 8.41 & 8.40 & 4.63 & 4.60 & 4.62 \\
\hline $\mathrm{T}_{3}: \mathrm{GA}_{3} 75 \mathrm{mg} \mathrm{l}^{-1}$ & 8.47 & 8.52 & 8.49 & 4.77 & 4.72 & 4.75 \\
\hline $\mathrm{T}_{4}: \mathrm{NAA} 25 \mathrm{mg} \mathrm{l}^{-1}$ & 8.67 & 8.89 & 8.78 & 4.60 & 4.59 & 4.59 \\
\hline $\mathrm{T}_{5}:$ NAA $50 \mathrm{mg} \mathrm{l}^{-1}$ & 9.24 & 9.27 & 9.25 & 4.67 & 4.74 & 4.71 \\
\hline $\mathrm{T}_{6}:$ NAA $75 \mathrm{mg} \mathrm{l}^{-1}$ & 8.79 & 8.52 & 8.66 & 4.74 & 4.77 & 4.76 \\
\hline $\mathrm{T}_{7}: \mathrm{GA}_{3} 25 \mathrm{mg} \mathrm{l}^{-1}+\mathrm{NAA} 25 \mathrm{mg} \mathrm{l}^{-1}$ & 8.28 & 9.61 & 8.95 & 4.65 & 4.65 & 4.65 \\
\hline $\mathrm{T}_{8}: \mathrm{GA}_{3} 25 \mathrm{mg} \mathrm{l}^{-1}+\mathrm{NAA} 50 \mathrm{mg} \mathrm{l}^{-1}$ & 9.43 & 9.45 & 9.44 & 4.67 & 4.52 & 4.59 \\
\hline $\mathrm{T}_{9}: \mathrm{GA}_{3} 25 \mathrm{mg} \mathrm{l}^{-1}+\mathrm{NAA} 75 \mathrm{mg} \mathrm{l}^{-1}$ & 8.74 & 8.63 & 8.68 & 4.68 & 4.59 & 4.64 \\
\hline $\mathrm{T}_{10}: \mathrm{GA}_{3} 50 \mathrm{mg} \mathrm{l}^{-1}+\mathrm{NAA} 50 \mathrm{mg} \mathrm{l}^{-1}$ & 9.81 & 9.38 & 9.60 & 4.62 & 4.67 & 4.65 \\
\hline $\mathrm{T}_{11}: \mathrm{GA}_{3} 75 \mathrm{mg} \mathrm{l}^{-1}+\mathrm{NAA} 75 \mathrm{mg} \mathrm{l}^{-1}$ & 8.46 & 8.40 & 8.43 & 4.78 & 4.70 & 4.74 \\
\hline $\mathrm{T}_{12}:$ Control & 8.10 & 8.22 & 8.16 & 4.76 & 4.38 & 4.57 \\
\hline Year Mean & 8.90 & 8.98 & 8.94 & 4.67 & 4.63 & 4.65 \\
\hline S. Em. \pm & 0.21 & 0.26 & 0.18 & 0.06 & 0.07 & 0.07 \\
\hline C.D. at $5 \%$ & 0.62 & 0.75 & 0.50 & 0.17 & 0.20 & NS \\
\hline C.V.\% & 4.10 & 4.93 & 4.54 & 2.12 & 2.58 & 2.36 \\
\hline YT: S. Em. \pm & & & 0.23 & & & 0.06 \\
\hline YT: C. D. at $5 \%$ & & & NS & & & 0.18 \\
\hline
\end{tabular}




\section{References}

1. Arteca RN. Plant growth substances: Principles and applications. CBS Publishers, New Delhi, 1996.

2. Chaudhary BR, Sharma MD, Shakya SM, Gautam DM. Effect of plant growth regulators on growth, yield and quality of chilli (Capsicum annuum L.) at Rampur, Chitwan. J. Inst. Agric. Anim. Sci. 2006; 27:65-68.

3. Gomatinayagam M, Anuradha VE, Zhao C, Ayoola GA, Jaleel CA, Annerselvam RP. ABA and $\mathrm{GA}_{3}$ affect the growth and pigment composition in Andrographis paniculata Wall. Frontiers Biol. China. 2000; 4:337-341.

4. Govind, Maji S, Kumawat R, Pal A, Kumar S, Saha S. Improvement of growth, yield and quality of garlic (Allium sativum L.) cv. G-282 through a novel approach. Bioscan. 2015; 10(1):23-27.

5. Ouzounidou G, Giannakoula A, Asfi M, Ilias I. Differential responses of onion and garlic against plant growth regulators. Pakistan J. Bot. 2011; 43(4):20512057.

6. Ouzounidu G, Ilias I. Hormone induced protection of sunflower photosynthetic apparatus against $\mathrm{Cu}$ toxicity. Biologia Plantarum. 2005; 49:223-228.

7. Ouzounidu G, Ilias I, Giannakoula A, Parthena P. Comparative study on the effects of various plant growth regulators on growth, quality and physiology of (Capsicum annum L.). Pakisthan J. Bot. 2010; 42(2):805814.

8. Patel MJ, Patel HC, Chavda JC. Influence of plant growth regulators and their application methods on yield and quality of onion (Allium cepa L.). Asian J. Hort. 2010; 5(2):263-265.

9. Purohit SS. Growth Regulators, Plant Physiology, National Book Trust, New Delhi, 2007, 32-46.

10. Shah SH, Ahmad I, Saimullah. Respones of Nigella sativa to foliar application of gibberellic acid and kinetin. Biologia Plantarum, 2007; 51:563-566.

11. Tiwari RS, Ankur A, Sengar SC. Effect of bioregulators on growth, bulb yield, quality and storability of onion cv. Pusa Red. Indian J. Pl. Physiol. 2003; 8(4):411-413.

12. Weaver RJ. Plant Growth Substances in Agriculture. W. H. Freeman and Co., San Francisco, 1972, 339p. 